\title{
Ruptura del bronquio principal contralateral durante lobectomía por cirugía toracoscópica videoasistida uniportal, y tres casos de reparación exitosa
}

\author{
Zhilin Luo Tianhu Wang Hong Zhang \\ Departamento de Cirugía Torácica, Tercer Hospital Afiliado de la Universidad Médica Chongqing, Chongqing, China
}

\section{Palabras clave}

VATS uniportal · Ruptura bronquial · Reparación

\section{Resumen}

Antecedentes: Nuestro objetivo es discutir el tratamiento para la ruptura del bronquio principal contralateral durante una lobectomía por cirugía toracoscópica videoasistida uniportal (VATS uniportal).

Presentación de casos: Analizamos los datos clínicos de tres casos de ruptura del bronquio principal contralateral durante la VATS uniportal. La reparación quirúrgica se realizó inmediatamente bajo la VATS uniportal durante la operación; como resultado, tres casos de ruptura bronquial se repararon exitosamente, y se continuó hasta completar la lobectomía y la disección de nódulo linfático sistémico. Los casos se reexaminaron una semana después, y no se en- contró fístula en la tráquea ni en los bronquios por broncoscopía con fibra óptica. El rango de permanencia de la sonda pleural fue 6-9 días, y la estancia hospitalaria fue de 8-10 días. No se observaron anormalidades en la radiografía torácica cuando las tres pacientes regresaron al hospital un mes después de la operación para una segunda revaloración.

Conclusiones: Se recomienda la reparación quirúrgica instantánea para el tratamiento de la ruptura bronquial en la cirugía torácica. Reparar un desgarre bronquial con VATS uniportal es seguro y factible.

(c) 2021 Luo, Wang, Zhang

\section{Antecedentes}

Para facilitar la cirugía torácica, se requiere la colocación de un tubo endotraqueal de doble lumen, con la finalidad de garantizar el aislamiento de los pulmones. La ocurrencia de lesiones traqueobronquiales (LTB) durante el proceso es un problema muy difícil de tratar, que incluso puede ser mortal. Reportes previos en la literatura indican que la incidencia de lesiones en las vías respiratorias causadas por intubación endotraqueal de doble lumen es muy baja, aproximadamente $0.2 \%$ [1]. La lesión en las vías respiratorias es una complicación rara, y en ocasiones no es fácil encontrar un desgarre bronquial relativamente pequeño asociado con el procedimiento. Entre las causas de la lesión se incluye la falta de anestesiólogos experimentados, lesiones accidentales asociadas con la operación del cirujano, malformaciones anatómicas de los propios bronquios traqueales, la intubación repetida y el inflado excesivo del globo. En este trabajo, nuestro objetivo es discutir el tratamiento para la ruptura del bronquio principal contralateral durante una lobectomía por VATS (video-assisted thoracoscopic surgery, cirugía toracoscópica videoasistida) uniportal.

\section{Presentación de casos}

En este trabajo, los autores reportan la ocurrencia y el tratamiento de tres casos de ruptura bronquial en 1075 casos de cirugía 
pulmonar de junio de 2016 a julio de 2019 en nuestro hospital (el Tercer Hospital Afiliado de la Universidad Médica Chongqing, Chongqing, China).

Las tres pacientes fueron de sexo femenino, con edad de 54 a 74 años, altura de $150 \mathrm{a} 163 \mathrm{~cm}$ y peso de 59 a $74 \mathrm{~kg}$. A las tres pacientes se les diagnosticaron lesiones que invadían el espacio pulmonar en un examen preoperatorio mediante tomografía computarizada (TC). El diagnóstico patológico de la biopsia por punción percutánea se confirmó como cáncer periférico de pulmón: en el lóbulo inferior derecho en dos pacientes, y en el lóbulo superior derecho en una paciente. No hubo indicaciones de metástasis distante con PET-TC en el examen preoperatorio de las tres pacientes; no hubo contraindicaciones quirúrgicas en la función pulmonar o cardiaca, en el electrocardiograma ni en la angiografía coronaria por TC; los vasos sanguíneos pulmonares, la tráquea y los bronquios podían seccionarse, como se apreció en la TC torácica con realce, y no se observaron anormalidades en la tráquea o los bronquios mediante broncoscopía preoperatoria con fibra óptica. Con respecto a las comorbilidades, había una paciente complicada con artritis reumatoide, quien recibió agentes inmunosupresores de largo plazo; una paciente padecía enfermedad cardiaca coronaria, y otra se reportó sin comorbilidades. En las discusiones preoperatorias con un equipo multidisciplinario (EMD) se identificó que la cirugía estaba indicada en las tres pacientes y no había contraindicaciones quirúrgicas.

Los anestesiólogos para los tres casos tienen más de 20 años de experiencia. Se indujo anestesia general con Sufentanilo, Propofol, bromuro de Rocuronio y Etomidato. Antes de la intubación, tras considerar cuidadosamente la altura y el peso de las pacientes y luego de medir el diámetro bronquial mediante imágenes de la TC torácica preoperatoria, se preparó un tubo bronquial de doble lumen del No. 35 para dos pacientes, y del No. 37 para la otra. Una vez que la punta del tubo hubo pasado a través de las cuerdas vocales, se retiró la sonda, el tubo se giró 90 grados en sentido antihorario y se hizo avanzar. El proceso de intubación transcurrió sin incidentes y no se encontró resistencia durante la colocación. Finalmente, la posición del tubo se confirmó por broncoscopía con fibra óptica. Tras completar la intubación, las pacientes adoptaron la posición lateral izquierda. Luego de cambiar la posición corporal, la posición del tubo se confirmó de nuevo, sin sangrado en la vía respiratoria; en este punto, la intubación era satisfactoria. Debido a que el diagnóstico patológico preoperativo identificó claramente cáncer de pulmón, en las tres pecientes se realizó lobectomía inferior derecha y disección de nodos linfáticos sistémicos con VATS uniportal. Para la operación, las pacientes adoptaron la posición lateral izquierda, y las incisiones, con una longitud aproximada de $3 \mathrm{~cm}$, se localizaron en el cuarto espacio intercostal del lado derecho, en la línea axilar anterior o la línea axilar media. La disección de los nódulos linfáticos abarcó las estaciones $2,4,7,8$, 9, 10 y 11. La estación 7 se seccionó de la siguiente manera: después de que el pulmón derecho colapsó, el apósito se sujetó con una pinza oval para presionar el pulmón hacia el mediastino anterior, y así revelar completamente la estructura mediastinal posterior. La pleura mediastinal posterior se abrió entonces con

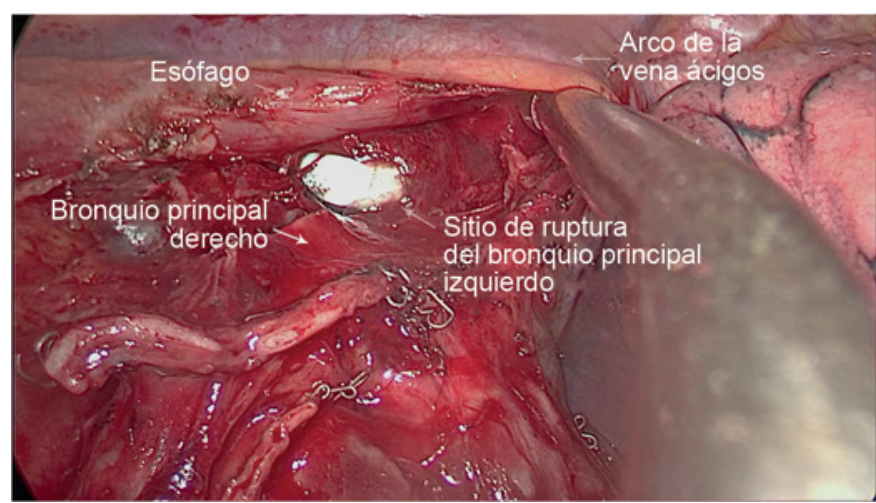

Fig. 1. Sitio de ruptura en el bronquio principal izquierdo antes de la reparación.

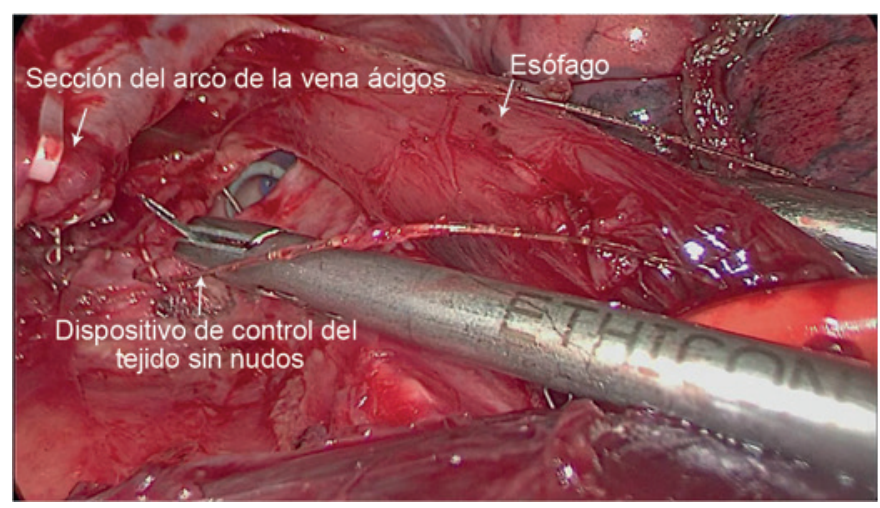

Fig. 2. Durante el proceso de reparación.

una cuchilla ultrasónica para revelar la estructura subcarinal y proceder a la disección de la estación 7. En las tres pacientes se encontró una ruptura longitudinal del bronquio principal izquierdo, con una longitud de $3 \mathrm{~cm}$, mientras el globo de la intubación traqueal aún se encontraba dentro, luego de la abertura de la pleura mediastinal posterior. Las pacientes tenían un enfisema obvio bajo el mediastino posterior, pero el globo de la intubación se hallaba intacto (Fig. 1).

Proceso de reparación: Se continuó la reparación del bronquio principal izquierdo mediante VATS uniportal mediante la incisión para el puerto original. La estructura subcarinal se disoció completamente para revelar los bronquios principales, izquierdo y derecho. El arco de la vena ácigos se disecó usando una grapadora lineal, y posteriormente el esófago se disoció completamente cerca de la carina y se retiró hacia el mediastino anterior. Luego de revelar por completo el sitio de ruptura del bronquio principal izquierdo, se suturó continuamente con espiral Stratafix 3-0 sin nudos (Fig. 2, 3). Los siguientes puntos deben tenerse en cuenta al suturar: 1. Sutúrese desde el extremo distal del sitio de ruptura hacia el extremo proximal; 2. Elija espiral Stratafix 3-0 de 1/2 C para acortar el tiempo de la operación tanto como sea posible; 3 . Durante el proceso de sutura, ponga atención especial a la succión de sangre y exudado en el sitio operado del tórax, de manera que no fluya dentro del bronquio contralateral a través de la ruptura 


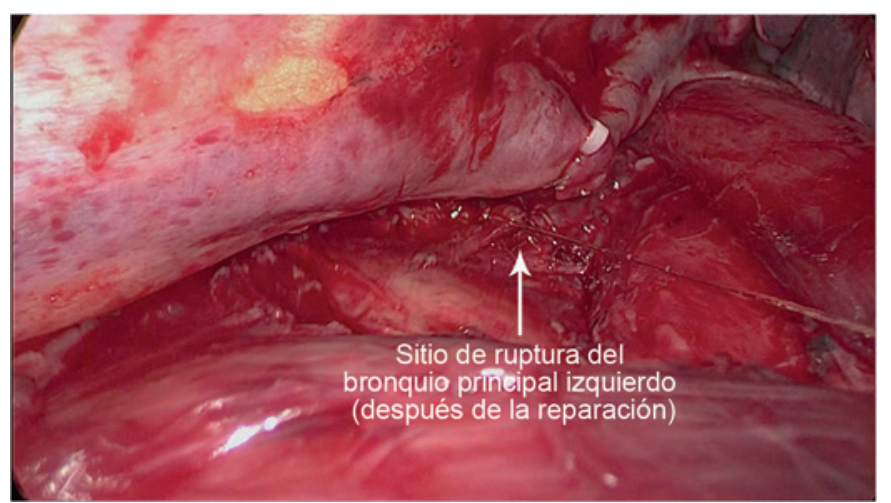

Fig. 3. Después de la reparación. No se detectó filtración de aire en la prueba con agua.

bronquial; 4. Al suturar, trabaje en cooperación estrecha con el anestesiólogo; esto es, cuando inserte la aguja, el tubo traqueal debe retraerse $0.5 \mathrm{~cm}$ para dejar espacio para la sutura; tras remover la aguja, hágase avanzar el tubo traqueal hasta que el extremo distal del tubo endotraqueal bloquee completamente la ruptura bronquial. Esto no sólo satisface el requerimiento para la sutura, sino que también asegura la ventilación del pulmón contralateral y el colapso del pulmón en el lado operado. Luego de completar la sutura, no se observó filtración alguna de aire durante la prueba con agua y el inflado del pulmón. Después de completar la reparación del bronquio principal izquierdo, se continuó la lobectomía inferior derecha y la disección de nódulos linfáticos con la VATS uniportal. El tiempo total de la operación varió entre 203 y $256 \mathrm{~min}$ en las tres pacientes, y la pérdida de sangre varió de 110 a $175 \mathrm{ml}$.

Los signos vitales de las pacientes se mantuvieron estables durante la operación. En ese mismo día, se transfirió a las pacientes a la Unidad de Cuidados Intensivos (UCI) para monitoreo y tratamiento postoperatorio, y se les trasladó a la sala general al siguiente día. Una semana después de la operación, se encontró congestión y edema en la mucosa bronquial, pero no se observó fístula (Fig. 4). El tiempo de permanencia del tubo torácico fue de 6-9 días, y la estancia hospitalaria fue 8-10 días. No se observaron anormalidades en una revaloración con radiografía torácica medio mes después del alta médica (Fig. 5).

\section{Discusión}

La intubación endotraqueal de doble lumen, esencial en la mayoría de las cirugías torácicas actuales, permite aislar los pulmones, de manera que el lado quirúrgico pueda colapsar y facilitar la operación. Sin embargo, antes de la intubación, el anestesiólogo debe observar cuidadosamente los resultados de la broncoscopía preoperatoria, e identificar rasgos anatómicos anómalos en las vías respiratorias o estenosis en dichas vías en la TC torácica. Cabe señalar la importancia de elegir un tubo traqueal con diámetro apropiado, y moverlo con suavidad durante la intubación para reducir el riesgo de lesión traqueal causada por una intubación violenta. Después de

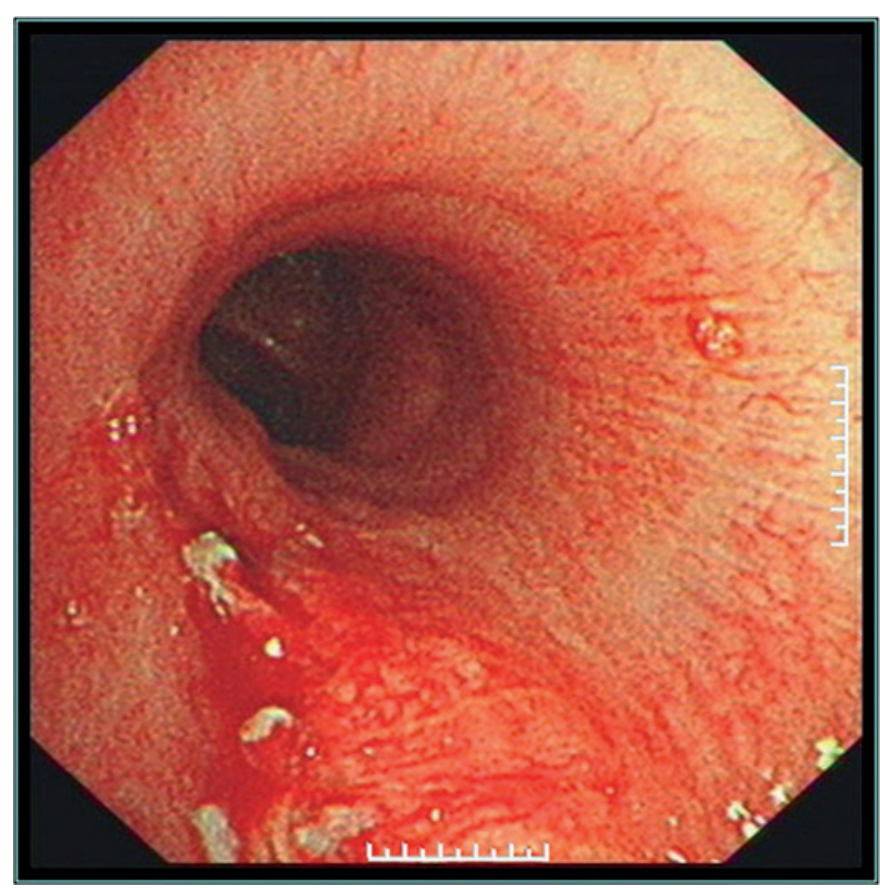

Fig. 4. Bronquio principal izquierdo bajo broncoscopía con fibra óptica una semana después de la operación.

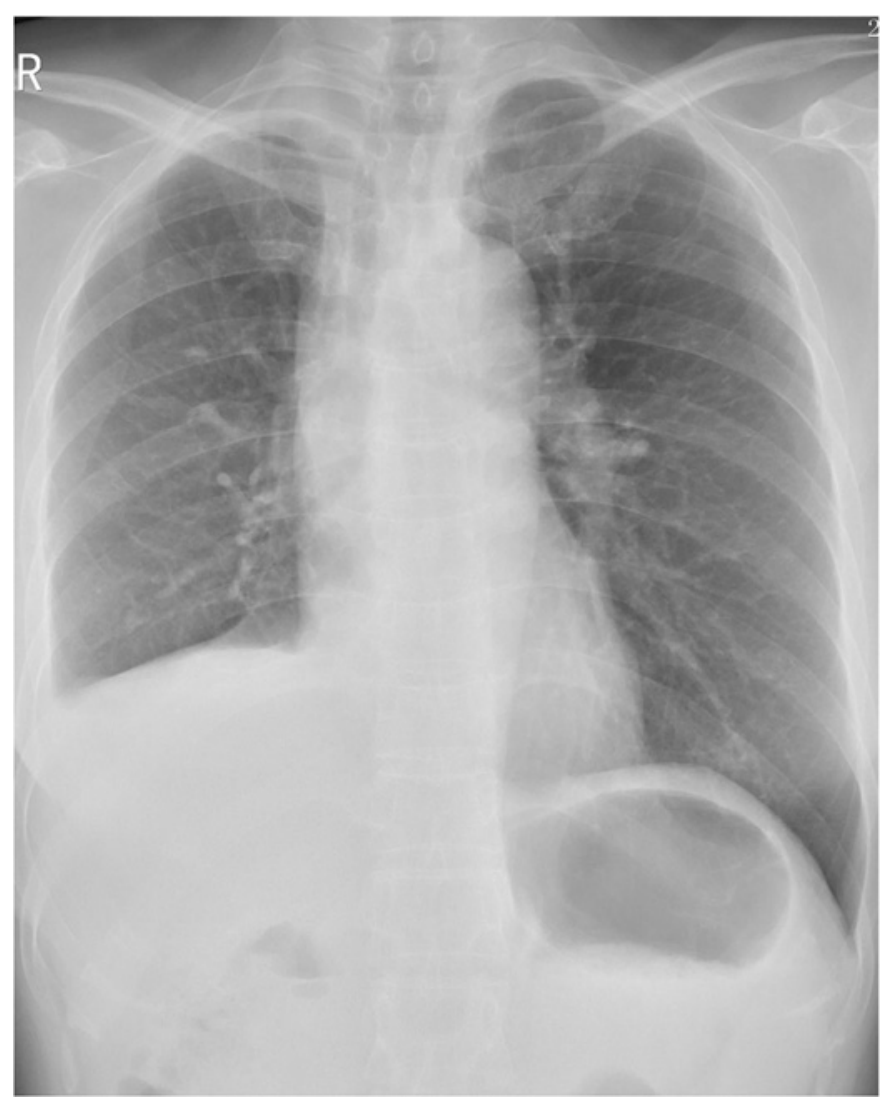

Fig. 5. Revaloración con radiografía torácica medio mes después del alta médica. No se observaron anormalidades. 
intubar, es recomendable tratar de inflar la manga bronquial con el menor volumen para lograr el aislamiento del pulmón. Al mismo tiempo, si es necesario, debe monitorearse la presión en la manga bronquial para mantenerla $<35-40 \mathrm{cmH}_{2} \mathrm{O}$ [2].

$\mathrm{Al}$ completar la intubación con un tubo endotraqueal de doble lumen, hay muchas posibles causas para una ruptura traqueobronquial (incluyendo factores iatrogénicos como lesiones accidentales provocadas por anestesiólogos o cirujanos, además de malformaciones anatómicas de los propios bronquios traqueales) que deben evitarse durante el tratamiento clínico. Se considera que los tres casos de ruptura intraoperatoria del bronquio principal izquierdo reportados en este trabajo están relacionados con el ajuste repetido de la posición de la intubación traqueal debido a un mal colapso intraoperatorio del pulmón, aunque no se excluyen factores como una presión excesiva en el globo. Una ruptura menor asociada con una LTB podría no encontrarse a tiempo durante la operación; sin embargo, si hay un grado considerable de enfisema subcutáneo o mediastinal, sonidos respiratorios disminuidos, dificultad respiratoria, etc., un examen por TC torácica, broncoscopía con fibra óptica o una técnica relacionada debe realizarse de manera oportuna para confirmarla. Desde luego, se recomienda la reparación quirúrgica instantánea para el tratamiento de una LTB identificada durante la operación.

Se ha confirmado que la VATS, en especial la VATS uniportal, es tan efectiva para lograr la resección de carcinoma pulmonar como la cirugía toracoscópica de dos puertos y la cirugía abierta, pero con una herida aún menor [3,4]. Si ocurre una ruptura bronquial durante la VATS uniportal, la opción preferida es repararla desde el puerto único. Sin embargo, debe elegirse una aguja apropiada cuando se repare un desgarre bronquial con una VATS uniportal, debido al espacio de operación más estrecho, y debe elegirse sutura sin nudos siempre que sea posible, para reducir el tiempo de operación. Al mismo tiempo, se requiere la cooperación estrecha de un anestesiólogo para asegurar el colapso del pulmón en el lado quirúrgico y una buena ventilación del pulmón contralateral. Si resulta difícil para el operador trabajar con una VATS uniportal debido a su experiencia o sus habilidades quirúrgicas, puede recomendarse la apertura de un puerto adicional o transferir la tarea a una toracotomía, porque la reparación exitosa y oportuna de un desgarre bronquial es de la mayor importancia.
Para los pacientes con reparación de ruptura traqueobronquial, recomendamos: 1 . Si el paciente presenta riesgos nutricionales, se requiere dar soporte nutricional adecuado; 2 . Debe reexaminarse oportunamente una radiografía torácica para evaluar la situación de la reexpansión pulmonar; 3. Debe realizarse una broncoscopía con fibra óptica de manera oportuna para mostrar la condición de la vía respiratoria después de la sutura, e identificar el desgarre; 4. Debe pedirse a los pacientes que fuercen la tos, para facilitar el drenaje de esputo; si es necesario, puede realizarse succión de esputo bajo broncoscopía con fibra óptica, para garantizar la permeabilidad de la vía respiratoria, además de ajustar oportunamente los antibióticos administrados de acuerdo con los resultados del cultivo de esputo, parámetros sanguíneos rutinarios, procalcitonina y otros indicadores; 5 . Para pacientes con diabetes, debe controlarse la glucosa plasmática, si es necesario; para asistencia, debe consultarse al departamento de endocrinología.

\section{Conclusiones}

1. Se recomienda la reparación quirúrgica instantánea para el tratamiento de ruptura bronquial en la cirugía torácica. 2. Es seguro y factible reparar un desgarre bronquial con VATS uniportal.

\section{Conflictos de interés}

Los autores declaran que no existen conflictos de interés potenciales con respecto a la investigación, la autoría o la publicación de este artículo.

\section{Información sobre licencias}

Zhilin Luo, Tianhu Wang, Hong Zhang: Rupture of contralateral mainstem bronchus during uniportal video-assisted thoracoscopy surgery lobectomy and 3 successful cases of repair. J Cardiothorac Surg. 2021 May 13;16(1):129 (DOI: 10.1186/s13019-021-01507-w). ${ }^{\circ} 2021$ Los Autores (traducción; abreviaturas, agradecimientos, contribuciones de los autores, financiamiento, disponibilidad de datos y materiales, aprobación ética y consentimiento para participar, consentimiento para publicación, nota del editor abreviadas), protegido por CC BY 4.0 (https://creativecommons.org/ licenses/by/4.0/deed.es).

\section{Referencias}

Las referencias están disponibles en www.karger.com/Article/Fulltext/ 000519854 . 